\title{
Diseño y Validación de un Cuestionario de Percepción del Aprendizaje a través del Feedback entre Iguales en Educación Superior
}

\author{
Design and Validation of a Questionnaire about Learning Perception through Peer Feedback in \\ Higher Education
}

\author{
Angelina Sánchez-Martí1 ${ }^{1}$, José Luís Muñoz Moreno² y Georgeta Ion $^{3}$
}

\begin{abstract}
Resumen
A pesar de que el feedback entre iguales es una estrategia habitualmente vinculada a la evaluación, con gran impacto sobre el aprendizaje y desarrollo competencial del estudiante, existen pocas experiencias en las que se vincule al aprendizaje, y pocas aportaciones se fijan en analizar sus efectos. Por ende, este artículo presenta el diseño y validación de un cuestionario para analizar los efectos de dar feedback en el proceso de aprendizaje de los estudiantes universitarios de Ciencias de la Educación. Coherente con la teoría de la construcción social del conocimiento, se estructura inicialmente en torno a cuatro dimensiones: desarrollo cognitivo y metacognitivo, competencias sociales, competencias profesionales y aspectos socioafectivos e intrapersonales. El cuestionario ha sido autoadministrado a una muestra de 425 estudiantes de primer curso de la Universitat Autònoma de Barcelona. Los resultados del análisis nos llevan a proponer un instrumento final de 20 ítems y tres variables latentes que presenta unos adecuados índices de fiabilidad y validez.
\end{abstract}

Palabras clave: evaluación, feedback entre iguales, educación superior, instrumento de evaluación, validación

\begin{abstract}
Although peer feedback is a strategy usually linked to assessment, with a great impact on the student's learning and competence development, there are still few experiences in which it is linked to learning, and few contributions are set to analyse its effects. Therefore, this paper presents the design and validation process of a questionnaire to analyse the effects of giving peer feedback on the learning process of university students of Education Sciences. Consistent with the theory of the social construction of knowledge, it is initially structured around four dimensions: cognitive and metacognitive development, social competencies, professional competences, and socio-affective and intrapersonal aspects. The questionnaire was selfadministered to a sample of 425 first-year students of the Universitat Autònoma de Barcelona. The results of the analysis lead us to propose a final questionnaire of 20 items and three latent variables with adequate reliability and validity indexes.
\end{abstract}

Keywords: assessment, peer feedback, higher education, evaluation tool, validation

\footnotetext{
${ }^{1}$ Doctora en Educación y Sociedad y Licenciada en Psicopedagogía por la Universidad de Barcelona. Investigadora Postdoctoral. Universitat Autònoma de Barcelona. Departamento de Pedagogía Sistemática y Social. Plaça del Coneixement. Edificio G6, Despacho 173. Campus de la UAB, 08193 Bellaterra (Cerdanyola del Vallès), Barcelona, España. Correo: angelina.sanchez@uab.cat. Tel.: +34935811411

${ }^{2}$ Doctor en Calidad y Procesos de Innovación Educativa y Licenciado en Pedagogía. Profesor Agregado Interino. Universitat Autònoma de Barcelona. Departamento de Pedagogía Aplicada. Plaça del Coneixement. Edificio G6, Despacho 267. Campus de la UAB, 08193 Bellaterra (Cerdanyola del Vallès), Barcelona, España. Correo: joseluis.munoz@uab.cat. Tel.: +34 935811417

${ }^{3}$ Doctora en Ciencias de la Educación por la Universidad de Barcelona y Licenciada en Psicología y Ciencias de la Educación por la Universidad de Bucarest. Profesora Agregada Interina. Universitat Autònoma de Barcelona. Departamento de Pedagogía Aplicada. Plaça del Coneixement. Edificio G6, Despacho 248. Campus de la UAB, 08193 Bellaterra (Cerdanyola del Vallès), Barcelona, España. Correo: georgeta.ion@uab.cat. Tel.: +34 935811620

Revista Iberoamericana de Diagnóstico y Evaluación - e Avaliação Psicológica. RIDEP · No53 · Vol.4 · 113-128 · 2019

ISSN: 1135-3848 print /2183-6051online
} 


\section{Introducción}

En las últimas décadas, los estudios sobre el papel de los diferentes agentes educativos en el proceso de formación de las competencias profesionales de los estudiantes han ido en aumento (Pereira, Assunção, \& Niklasson, 2016). En ellos, la evaluación emerge como un contexto favorable al aprendizaje en el que interactúan diferentes actores y que implica una gran variedad de métodos y estrategias (Nicol, Thomson, \& Breslin, 2014). La evaluación entre iguales representa, en este contexto, una de las estrategias vinculadas con la evaluación formativa, orientada hacia el desarrollo competencial y el aprendizaje (Cano, 2014), comprometiendo a los estudiantes como agentes activos en la evaluación. Sin embargo, sistemas como la evaluación entre iguales, la coevaluación o la autoevaluación, todavía tienen poca presencia en la formación universitaria (San Martín, Jiménez, \& Jerónimo, 2016).

Cuando se analiza el ciclo de la evaluación desde la creación de la tarea de aprendizaje -pasando por la producción, evaluación y retorno al estudiante-, se constata claramente que uno de los aspectos con mayor impacto sobre el sistema y que resulta fundamental en el aprendizaje futuro de los estudiantes es el feedback que éstos reciben (Ajjawi \& Boud, 2018). De hecho, la revisión sistemática realizada por Hattie (1987), con más de 800 metaanálisis, destaca el feedback como uno de los elementos que más impacta en el aprendizaje del estudiante. Las revisiones realizadas por Black y Wiliam (1998) corroboran también que el feedback tiene el potencial de ser uno de los procesos con mayor efecto positivo sobre la mejora del aprendizaje desde la perspectiva docente. El feedback ha sido así considerado el séptimo elemento de los once condicionantes más importantes que apoyan el aprendizaje (Gibbs \& Simpson, 2002).

La mayor parte de la literatura especializada sobre la temática repara en los distintos condicionantes que permiten mejorar la provisión del feedback (Carless \& Boud, 2018), y pocas aportaciones se fijan en analizar los efectos que tiene sobre los diversos aspectos vinculados al aprendizaje y a las competencias del estudiante
(Winstone, Nash, Parker, \& Rowntree, 2017). Además, una parte importante de los estudios realizados sobre la temática se centran en el feedback ofrecido por el profesorado (Winstone et al., 2017) y son menores las aportaciones que consideran las potencialidades del feedback entre iguales (Panadero, 2016).

Por ende, este artículo tiene como objetivo proponer un modelo de análisis de los efectos del feedback entre iguales sobre la variedad de componentes del aprendizaje del estudiante, ofreciendo un diseño integrado y multinivel, compuesto por elementos de índole cognitiva, interpersonal y profesional. Se facilita de esta manera una constelación de variables vinculadas al desarrollo cognitivo, metacognitivo, afectivo, social y profesional del estudiante.

\section{Dar feedback entre iguales como estrategia formativa}

El feedback entre iguales es aquel proceso de retroalimentación proporcionado por estudiantes con el mismo estatus. Se puede considerar como una forma de evaluación formativa, complementaria a los comentarios del profesorado (Topping, 1998) y una forma de aprendizaje colaborativo (van Gennip, Segers, \& Tillema, 2010). También es una estrategia muy efectiva para mejorar el aprendizaje (Gielen, Peeters, Dochy, Onghena, \& Struyven, 2010), la autorregulación del mismo (Cabrera \& Mayordomo, 2016) y la calidad de las contribuciones propias y de otros (Molloy \& Boud, 2015). Transita así hacia la perspectiva de la participación del estudiante en el aprendizaje y desde la colaboración (Winstone et al., 2017), posibilitando crear opiniones, posicionamientos y preferencias que influyen en el estudiante ante su capital situación en el proceso formativo.

El feedback entre iguales pertenece a una visión de la enseñanza que prioriza la naturaleza formativa y continuada de la evaluación, facilita el aprendizaje del estudiante y promueve el desarrollo de futuros profesionales activos, responsables y reflexivos (Quinton \& Smallbone, 2010). De este modo, deriva del constructivismo social, mediante la construcción conjunta del conocimiento a través del discurso y la interactividad, y lleva implícitas las habilidades comunicativas y sociales (Vygotsky, 1978). 
Además, se entiende como un proceso derivado del modelo piagetiano de conflicto cognitivo, porque involucra a estudiantes con un mismo estatus (Ibarra Sáiz et al., 2012), pero con competencias diferentes, lo que implica un proceso de negociación de significados y conocimiento mutuo (Zhu \& Carless, 2018).

El feedback representa también un proceso de intercambio de mensajes y significados entre los agentes implicados $\mathrm{y}$, siguiendo el modelo interpersonal de comunicación (Johnson \& Johnson, 1994), incluye variables vinculadas al transmisor del mensaje, al receptor, al mensaje mismo y al contexto en el cual se desarrolla el proceso comunicativo. Tomando en cuenta estos elementos, parece evidente que el proceso de aprendizaje se relaciona intrínsecamente con el momento de dar y de recibir el feedback.

La literatura que analiza el rol del feedback en el aprendizaje del estudiante, se focaliza fundamentalmente en los beneficios de recibirlo (Orsmond \& Merry, 2013), siendo más limitados los estudios sobre el impacto que éste tiene sobre aquellos que lo ofrecen. Partiendo de esta premisa, y siendo conscientes de los beneficios del feedback sobre los propios emisores (Ion, Sánchez Martí, \& Agud Morell, 2018), este estudio se centra basicamente en tal situación, ya que la concreción de dar feedback persigue una participación activa del estudiante en su proceso de aprendizaje, así como frente a la evaluación (Neugebauer, Ray, \& Sassenberg, 2016; Panadero, Jonsson, \& Strijbos, 2016a). Cuando el estudiante da feedback está aprovechando una oportunidad para aprender, que incide muy positivamente en la evaluación por cuanto se desarrolla su capacidad para realizar juicios fundamentados (Carless, 2016).

El hecho que el estudiante ofrezca feedback enlaza con el asesoramiento, la revisión, la emisión de comentarios y aclaraciones, el diagnóstico de errores, la identificación de lagunas en el conocimiento, la delimitación de disfunciones en las actividades o la reflexión formativa (Harris \& Brown, 2013; Topping, 1998). Así, se da soporte a la consolidación, el refuerzo y la profundización de los aprendizajes (Topping, 2009; Tsivitanidou, Zacharia, \& Hovardas, 2011), y se da responsabilidad y protagonismo ante el conocimiento para que siga avanzando en su formación y la de sus iguales (Ajjawi \& Boud, 2018).

En un proceso de feedback entre iguales, la examinación y valoración del trabajo académico de otros, el reconocimiento de sus fortalezas y debilidades y la puntualización de áreas de mejora, conducen al aprendizaje del estudiante porque implica un compromiso cognitivo superior: aplicar criterios, localizar problemas y sugerir soluciones (Nicol et al., 2014). Sin embargo, dar feedback no queda exento de encontrarse con algunas barreras (Telio, Ajjawi, \& Regehr, 2015) y, en consecuencia, conviene insistir en acompañar y animar al estudiante a generar e integrar el feedback, impulsando su capacidad crítica y creando un clima proclive (Carless, 2016).

\section{Los efectos de dar feedback entre iguales sobre el aprendizaje}

El valor formativo de dar feedback ha sido analizado desde múltiples enfoques y variables. Desde enfoques más cualitativos -centrados especialmente en la percepción de la satisfacción del estudiante- hasta otros más cuantitativos y vinculados a variables metacognitivas (Roth, Ogrin, \& Schmitz, 2015). El análisis se ha fijado en detectar los factores que inciden en su eficiencia, como el tipo de tarea, el tiempo, el compromiso con el proceso y la práctica (Espasa, Guasch, Mayordomo, Martínez-Melo, \& Carless, 2018; Topping, 1998).

Participar en un proceso de dar feedback entre iguales, en primer lugar, influye en el desarrollo cognitivo y metacognitivo de los estudiantes (Cano \& Pons, 2019). Esto tiene que ver con que el estudiante encuentre sentido a la experiencia y demostración del conocimiento, haciendo referencia a las estrategias que emplea para aprender. El desarrollo cognitivo se alinea con el conocimiento individual de las cogniciones del estudiante y su habilidad para controlarlas. Las principales habilidades cognitivas que activa quien da feedback se relacionan con atender, comprender, elaborar, recuperar, etc., mientras que las habilidades metacognitivas enlazan con dirigir, evaluar y modificar el propio aprendizaje (Carless, 2015; Molloy \& Boud, 2015). Por consiguiente, dar feedback plantea la necesidad de resumir, reestructurar cognitivamente, confirmar o 
desestimar diferentes elementos cognitivos, generalizar el conocimiento o transferirlo a otras situaciones. Algunos de estos elementos son más visibles y otros más latentes (David Boud \& Falchikov, 2006), pero todos inciden en el desarrollo futuro del estudiante.

En segundo lugar, aprender cómo ofrecer críticas, y justificar posiciones y decisiones tomadas, sirve al desempeño de habilidades sociales y asertivas (Carless \& Chan, 2017) y de habilidades comunicativas verbales y escritas (Neugebauer et al., 2016), propias del trabajo en equipo o la negociación. Las habilidades y competencias sociales están conectadas con las capacidades del estudiante que da feedback por percibir e interpretar la situación académica y el contexto formativo donde se encuentra, pero también con la utilización de recursos para contribuir oportunamente en la relación con sus iguales (Ortego, López, \& Álvarez, 2011). Las capacidades más recurrentes que se activan en el estudiante que proporciona feedback suelen ser la confianza, la sociabilidad, el apoyo o la colaboración.

En tercer lugar, la implicación del estudiante en procesos de evaluación también contribuye a la formación de sus competencias profesionales futuras (Marcoulides \& Simkin, 2010). Dar feedback favorece el desarrollo de estrategias de pensamiento reflexivas, críticas e independientes (Ibarra Sáiz et al., 2012), el conocimiento de distintas soluciones a diferentes situaciones problemáticas (Gibbs \& Simpson, 2002), el aumento de la autosuficiencia y la dirección del propio aprendizaje (Stefani, 1994), una mayor capacidad de diplomacia y negociación (Neugebauer et al., 2016) o el fomento del pensamiento e incremento de la confianza en el aprendizaje (Carless, 2015). Todas estas facilitan, sin duda, el aprendizaje permanente del estudiante a lo largo y ancho de la vida.

Por último, dar feedback entre iguales guarda una relación directa con los elementos socioafectivos e intrapersonales del aprendizaje (Paoloni \& Rinaudo, 2009), y apoya el sentimiento de pertinencia, la responsabilidad personal y la motivación (Carless \& Chan, 2017). Acentúa el esfuerzo y el progreso personal, brinda alternativas de acción para evolucionar en las tareas (Alexander, 2006), transmite informaciones de la calidad de las aportaciones, viabiliza la autoevaluación para conseguir el objetivo propuesto o ampara la conciencia de qué supone aprender (Alonso, 2000), entre otros aspectos. Los efectos motivacionales del feedback refuerzan la perseverancia del estudiante ante la tarea y le ayudan a mantener los esfuerzos para lograr sus metas (Carless, 2016). Por eso, es importante suministrar comentarios críticos y de elogio al desempeño de los iguales (Rowe, Wood, \& Petocz, 2008), y no tanto hacer contribuciones con efectos negativos. Además, aumenta la empatía y la confianza en uno mismo (Ion, Cano, \& Fernández, 2017) y ejercita las competencias socioafectivas que son imprescindibles en educación.

\section{Instrumentos para la evaluación del feedback entre iguales}

Para conocer el impacto que tiene el uso del feedback entre iguales en el aprendizaje y el desarrollo competencial del estudiante, así como para explicar su influencia en la adquisición de aprendizajes más profundos y reflexivos dentro de los procesos formativos universitarios (Gan, Nang, \& Mu, 2018), es necesario recurrir a la aplicación de instrumentos fiables y válidos.

No obstante, existe un espectro relativamente limitado de los instrumentos que analizan el feedback. Por un lado, el estudio del feedback entre iguales se focaliza sobre todo en el análisis de los tipos de feedback con mayor impacto sobre el aprendizaje (Dawson et al., 2018; Espasa et al., 2018). Por otro lado, se destacan dos posibles escenarios en cuanto al efecto del feedback sobre el aprendizaje del estudiante. El primero explora las experiencias con el feedback desde una perspectiva subjetiva, haciendo uso de instrumentos como las entrevistas individuales y grupales (Crichton \& Valdera Gil, 2015) o el análisis de experimentos con el estudiante ( $\mathrm{Li}$, Liu, \& Steckelberg, 2010). El segundo recurre al uso de cuestionarios que interrogan sobre diversos elementos del aprendizaje: la motivación del estudiante (Gan, Nang \& Mu, 2018), las concepciones sobre la evaluación (Fletcher, Meyer, Anderson, Johnston, \& Rees, 2012) o las competencias transversales (Neugebauer et al., 2016). 
En el contexto español, los trabajos recientes de Gallego, Quesada, Gómez y Cubero (2017), Santana-Bonilla, Jiménez, Pintor y Hernández (2017), Mauri, Ginesta y Rochera (2016) o Plana (2016) reflejan que la evaluación con feedback y la regulación del aprendizaje han adquirido importancia, pero su estudio es todavía incipiente y poco frecuente. Por eso, conviene seguir indagando acerca de la calidad y la utilidad del feedback que se ofrece, así como conocer el uso que los estudiantes hacen de la información recibida.

Para ello, conviene dotarse de herramientas conceptuales y metodológicas que favorezcan recoger información valiosa sobre los aspectos señalados y disponer de marcos de referencia para su análisis (Santana-Bonilla et al., 2017). Además, entre las principales herramientas metodológicas existentes, la mayoría tienden a medir una única dimensión conceptual, la cognitiva y metacognitiva (Espasa, 2009), por lo que es evidente la pertinencia de contribuir con nuevos instrumentos que respondan a una visión más holística y completa del feedback.

\section{Método}

El doble objetivo principal que persigue el trabajo realizado ha sido diseñar y validar un instrumento de medida de las estrategias de evaluación entre iguales mediante el feedback, con adecuados y aceptables niveles de fiabilidad y validez. Por eso, se ha llevado a cabo un estudio por encuesta.

\section{Instrumento}

Se diseñó un cuestionario autoadministrado online, desde la plataforma "SurveyMonkey" (https://www.surveymonkey.com/r/7H5SMB3), denominado Estrategias de Evaluación entre Iguales y Feedback (EAIF), para analizar la incidencia de los procesos de feedback entre iguales en el aprendizaje del estudiante.

Se tomó como referencia la teoría de la evaluación entre iguales, fundamentada en la idea de la construcción social del conocimiento mediante el discurso y la interactividad entre los agentes que ofrecen y reciben el feedback. Esta interacción se produce en cualquier tipo de contexto, incluido el que no supone una interacción cara a cara (Vygotsky, 1978). Además, la evaluación entre iguales supone implícitamente un estatus de igualdad entre agentes que poseen competencias diferentes, lo que nos acerca al modelo piagetiano del conflicto cognitivo. La corroboración entre estas perspectivas resulta de especial interés, especialmente en contextos de aprendizaje cooperativo, como es el caso de ámbito en el que nos fijamos.

A partir de aquí se formularon las categorías de análisis y se diseñaron los ítems, considerando los efectos que tiene la evaluación entre iguales en el aprendizaje del estudiante y partiendo del modelo propuesto por Topping (1998) que distingue entre los siguientes dominios: cognición y metacognición, afectividad, habilidades sociales y transferibles, y beneficios sistémicos. La dimensión de habilidades afectivas e interpersonales fue ampliada, siguiendo las contribuciones de Panadero (2016) sobre el rol de éstas en el desarrollo del estudiante y fue creada una dimensión más amplia de competencias profesionales desde la categoría definida por Topping (1998) dentro del marco de "beneficios sistémicos". Se incluyeron aquellos aspectos vinculados a la concepción que se tiene acerca de la evaluación como proceso formativo, considerando que representan competencias críticas para la formación de los futuros maestros (Panadero, Brown, \& Courtney, 2014). Las categorías se resumen a continuación (Tabla 1):

(1) Desarrollo cognitivo y metacognitivo del estudiante (resumir, reestructurar, contrastar y reflexionar).

(2) Habilidades y competencias sociales (el aprendizaje activo, la aceptación de críticas, la argumentación, trabajo en grupo y asertividad).

(3) Competencias profesionales futuras de los estudiantes actuales (competencias evaluativas vinculadas a la profesión docente, concepción acerca de la evaluación y percepción sobre las estrategias de evaluación).

(4) Desarrollo de aspectos afectivos e intrapersonales (responsabilidad, ansiedad, empatía y nivel de aceptación de los comentarios negativos).

El tipo de escalamiento de los ítems presenta una valoración numérica tipo Likert de 1 a 7 , 
donde 1 representa la menor valoración (nunca) y 7 la mayor valoración (siempre). El cuestionario completo incluye 64 ítems escalares que analizan la percepción del aprendizaje cuando se da feedback (32 ítems) y los mismos ítems analizando el aprendizaje cuando se recibe feedback (32 ítems).

Considerando los beneficios demostrados del feedback para aquellos que lo ofrecen (Ion, Sánchez-Martí, \& Agud, 2018; Kim, 2009), la validación del modelo confirmatorio aquí recogido se fija precisamente en los 32 ítems de "dar feedback".

\section{Muestra}

El cuestionario fue administrado a estudiantes universitarios de primer curso de una asignatura anual de Formación Básica Compartida ("Educación y Contextos Educativos") de la Facultad de Ciencias de la Educación de la Universitat Autònoma de Barcelona. La asignatura de 12 créditos ECTS es impartida en todas las titulaciones de grado que ofrece la Facultad: Educación Social, Pedagogía, Educación Infantil y Educación Primaria. La aplicación del cuestionario ha sido el resultado de la experiencia de innovación educativa y docente realizada para mejorar la provisión de feedback entre iguales y proponer tareas más vinculadas al desarrollo de las competencias de los estudiantes.

También se buscaba mejorar la implicación de los estudiantes en su proceso de aprendizaje, así como en el proceso de aprendizaje de sus compañeros, siendo conscientes de que pondrían en práctica habilidades que más adelante integrarán en su desarrollo profesional. Dado que todos los estudiantes fueron partícipes de esta experiencia, todos fueron invitados a contestar el cuestionario EAIF $(\mathrm{N}=550)$. La muestra participante fue de 463 estudiantes, el instrumento fue administrado presencialmente y el tiempo de aplicación osciló entre los 20 y los 30 minutos.

Para el análisis de datos se eliminaron aquellas respuestas que no estaban completas, de modo que finalmente se trabajó con una base de 425 estudiantes (error muestral del 2.3\%), de los que 64 son hombres (15.1\%) y 361 mujeres (84.9\%). La edad de los participantes oscila entre los 18 y los 41 años, siendo la media de 20.20 y la moda de 18 años. Por titulación de grado, la muestra queda compuesta por $43.5 \%$ estudiantes de Educación Primaria $(n=185), 24.9 \%$ de Educación Infantil ( $\mathrm{n}=106), 16.9 \%$ de Educación Social $(n=72)$ y $14.6 \%$ de Pedagogía $(n=62)$. El número de casos válidos que conforman la muestra es adecuado para los análisis estadísticos previstos (Lloret-Segura, Ferreres-Traver, Hernández-Baeza, \& Tomás-Marco, 2014).

\section{Análisis de datos}

El análisis de los datos se llevó a cabo siguiendo distintas fases. En la primera se estimó el poder discriminativo de los ítems a partir de un análisis univariante (medias, desviaciones, asimetría, curtosis, etc.) y bivariante (correlaciones de Pearson). En la segunda se llevó a cabo un análisis multivariante usando la técnica de análisis factorial exploratorio para identificar los factores latentes que permiten organizar los ítems que componen la escala. En la tercera, y última fase, se realizó un análisis factorial confirmatorio.

Optamos por el modelo de ecuaciones estructurales porque permite examinar simultáneamente relaciones de dependencia y evaluar la validez de los constructos medidos a partir del cuestionario diseñado. Concretamente, adoptamos la estrategia de modelización confirmatoria para evaluar la significación estadística del modelo propuesto. La intención última es corroborar la idoneidad de los indicadores seleccionados en la medición de los constructos definidos: desarrollo cognitivo y metacognitivo, habilidades y competencias sociales, competencias profesionales y, aspectos socioafectivos e intrapersonales. También se ha hecho un análisis de fiabilidad, en concreto el de consistencia interna de las escalas resultantes (alfa de Cronbach).

$\mathrm{Al}$ analizar la estructura interna, la muestra original se dividió en dos submuestras extraídas aleatoriamente (Lloret-Segura et al., 2014). La primera $(n=204)$ se utilizó para explorar la estructura factorial subyacente a los ítems calculando el análisis factorial exploratorio. La segunda submuestra $(n=221)$ se empleó para confirmar esa estructura usando el cálculo del análisis factorial confirmatorio.

La dimensionalidad del instrumento se examinó calculando la medida de adecuación 
muestral de Kaiser-Meyer-Olkin (KMO) y la prueba de esfericidad de Bartlett. Para determinar el ajuste de los modelos AFE y AFC se utilizaron los siguientes índices estadísticos: el índice relativo Tucker-Lewis (TLI), el índice comparativo de ajuste (CFI), el Índice de Bondad del Ajuste (GFI) y se estimó también el Error Cuadrático Medio de Aproximación (RMSEA). Para el ajuste de los modelos se consideran como aceptables valores TLI, CFI y GFI mayores a .9, y para RMSEA entre .05 y .08 (Calvo, 2017). El análisis de los datos se realizó con el apoyo de los programas estadísticos IBM SPSS v23.0 y AMOS v22.0.0.

\section{Resultados}

Seguidamente se destacan los principales resultados obtenidos en cada una de las fases descritas para el análisis y la validación del instrumento diseñado y la determinación del instrumento que emerge como definitivo.

\section{Análisis del poder discriminativo de los ítems}

Para determinar el poder discriminativo de los ítems y medir de la forma más precisa posible la cualidad del cuestionario con el menor número posible de elementos de medida, se verificó el cumplimiento de las condiciones de aplicabilidad del instrumento diseñado siguiendo las recomendaciones de trabajos previos (LópezAguado, 2010). De este modo, el análisis descriptivo de los ítems se hizo en base a la media, la desviación estándar y los índices de asimetría y curtosis (Tabla 1).

Los valores de los ítems presentan en su mayoría valores buenos, comprendidos en el rango -1,1 (Ferrando \& Anguiano-Carrasco, 2010). Se examinó la correlación elemento-total corregida para cada uno de los ítems tomando como válidos los superiores al valor .20 propuesto por Kline (2011). Asimismo, se examinó la multicolinealidad entre los ítems para descartar variables que fueran redundantes; es decir, correlaciones inter-ítem mayores de .95 . Únicamente el ítem 25 refleja un coeficiente dispar, de manera que se optó por eliminarlo en esta etapa de análisis.
Estructura latente del cuestionario: análisis factorial

\section{Análisis factorial exploratorio}

Tras la determinación de las cualidades psicométricas del instrumento, se analizó la validez del constructo a través de la exploración de la estructura factorial del conjunto de ítems total con la voluntad de identificar las variables subyacentes. Se ha utilizado el método de extracción de componentes principales con rotación Varimax porque se parte de la idea que no existen correlaciones entre los factores o estructuras que se suponen subyacentes $\mathrm{y}$, por consiguiente, son independientes unas de otras.

El índice KMO (Kaiser-Meyer-Olkin) arroja un valor de .969 , lo que informa de una correcta adecuación muestral. El índice de esfericidad de Bartlett $\left(\chi^{2}(465)=11241.831 ; p<.01\right)$ confirma la existencia de interrelaciones significativas entre las variables que informan de la adecuación de los datos a un modelo de análisis factorial. Así, se obtiene un modelo de tres factores que explican el $65,081 \%$ de la varianza (Tabla 2).

El primer factor $(n=13)$ contiene 8 ítems de desarrollo cognitivo y metacognitivo, y 5 ítems que inicialmente se habían asignado a las dimensiones de habilidades y competencias sociales y profesionales. La opción fue por mantener el nombre asignado inicialmente al factor "desarrollo cognitivo y metacognitivo", debido a que en este todos los ítems que se conjugan están relacionados con la organización y la dirección de procesos y actividades propias de estudio. El segundo factor $(\mathrm{n}=9)$ contiene 7 ítems que responden a elementos afectivos y 2 vinculados a las competencias profesionales. Se decidió nombrar a este segundo factor como "desarrollo intrapersonal e interpersonal", de acuerdo con los avances y las recomendaciones del cuerpo de conocimiento reciente sobre evaluación entre pares (Panadero, 2016). Finalmente, el tercer factor $(n=9)$ queda compuesto por 8 ítems de competencias sociales y profesionales y 1 que previamente se había asignado a las habilidades y competencias sociales. Como antes, dada la saturación de ítems de índole competencial, en este caso optamos por nombrar a este factor en un sentido genérico: desarrollo competencial. 
Tabla 1. Descriptivos y correlaciones ítem-test de los ítems del instrumento (“dar feedback")

\begin{tabular}{|c|c|c|c|c|c|c|c|c|}
\hline & & Items & $\mathrm{N}$ & Media & Desv. & Asimetría & Curtosis & $\begin{array}{c}\text { Correlación } \\
\text { ítem-test }\end{array}$ \\
\hline 1 & CPS* & $\begin{array}{c}\text { Ha sido útil para la mejora de las tareas de mi grupo } \\
\text { de trabajo. }\end{array}$ & 425 & 5.17 & 1.407 & -.625 & -.143 & .603 \\
\hline 2 & $\mathrm{COG}$ & $\begin{array}{c}\text { Me ha hecho dar más valor a las tareas de la } \\
\text { asignatura. }\end{array}$ & 425 & 4.61 & 1.522 & -.357 & -.468 & 689 \\
\hline 3 & INTRA & $\begin{array}{l}\text { Ha aumentado mi confianza en los demás } \\
\text { compañeros de equipo. }\end{array}$ & 425 & 4.17 & 1.543 & -.205 & -.493 & .620 \\
\hline 4 & $\mathrm{CPP}$ & Es una estrategia de aprendizaje útil para mí. & 425 & 5.14 & 1.455 & -.695 & .195 & .699 \\
\hline 5 & $\mathrm{CPP}$ & $\begin{array}{l}\text { Ha mejorado mi aceptación por parte de los } \\
\text { compañeros de grupo. }\end{array}$ & 425 & 4.35 & 1.533 & -.418 & -.344 & .627 \\
\hline 6 & $\mathrm{COG}$ & $\begin{array}{c}\text { Integrar los conocimientos de la asignatura en mis } \\
\text { conocimientos generales. }\end{array}$ & 425 & 5.04 & 1.377 & -.635 & .186 & .661 \\
\hline 7 & CPS & $\begin{array}{c}\text { Comparar mi trabajo con el de los demás } \\
\text { compañeros de clase. }\end{array}$ & 425 & 5.76 & 1.239 & -.993 & .878 & .355 \\
\hline 8 & COG & $\begin{array}{c}\text { Contrastar conocimientos previos con nuevos } \\
\text { conocimientos de la asignatura. }\end{array}$ & 425 & 5.26 & 1.324 & -.797 & .672 & .682 \\
\hline 9 & $\mathrm{CPP}$ & Mejorar mi capacidad comunicativa escrita y oral. & 425 & 4.87 & 1.448 & -.527 & -.102 & .716 \\
\hline 10 & COG & Aclarar dudas que tenía sobre la asignatura. & 425 & 4.62 & 1.520 & -.357 & -.413 & .661 \\
\hline 11 & COG & $\begin{array}{c}\text { Entender los criterios de evaluación y realizar la } \\
\text { tarea de acuerdo con estos. }\end{array}$ & 425 & 5.17 & 1.409 & -.659 & -.072 & .626 \\
\hline 12 & COG & Tomar conciencia de los objetivos de la asignatura. & 425 & 4.86 & 1.463 & -.485 & -.264 & .658 \\
\hline 13 & COG & Entender mejor las tareas realizadas. & 425 & 5.08 & 1.386 & -.750 & .359 & .709 \\
\hline 14 & COG & Entender mejor las tareas futuras. & 425 & 4.67 & 1.449 & -.413 & -.251 & .692 \\
\hline 15 & CPP & Mejorar mi competencia de trabajo en equipo. & 425 & 5.19 & 1.422 & -.746 & .164 & .705 \\
\hline 16 & CPS & Aprender más activamente. & 425 & 5.24 & 1.334 & -.733 & .345 & .758 \\
\hline 17 & $\mathrm{CPP}$ & Mejorar la capacidad de argumentación. & 425 & 5.11 & 1.384 & -.629 & .103 & .687 \\
\hline 18 & CPS & $\begin{array}{c}\text { Mejorar la comunicación con los compañeros de } \\
\text { grupo. }\end{array}$ & 425 & 5.08 & 1.458 & -.653 & -.007 & .648 \\
\hline 19 & $\mathrm{CPP}$ & Mejorar la capacidad de redactar mi discurso. & 425 & 5.07 & 1.390 & -.610 & .073 & .716 \\
\hline 20 & CPS & Evaluar mejor el trabajo de los compañeros. & 425 & 5.37 & 1.202 & -.561 & .084 & .674 \\
\hline 21 & INTRA & $\begin{array}{c}\text { Tomar conciencia de la responsabilidad de evaluar a } \\
\text { otros. }\end{array}$ & 425 & 5.73 & 1.243 & -.996 & .880 & .585 \\
\hline 22 & CPS & Evaluar mejor mi trabajo. & 425 & 5.46 & 1.179 & -.693 & .715 & .592 \\
\hline 23 & $\mathrm{CPP}$ & Mejorar la planificación de mis tareas. & 425 & 4.85 & 1.482 & -.543 & -.161 & .634 \\
\hline 24 & CPS & $\begin{array}{l}\text { Ver la importancia de utilizar diferentes estrategias } \\
\text { de evaluación para evaluar diferentes tareas. }\end{array}$ & 425 & 4.98 & 1.354 & -.406 & -.215 & .671 \\
\hline 25 & INTRA & Me ha generado ansiedad. & 425 & 2.74 & 1.761 & .746 & -.561 & -.069 \\
\hline 26 & INTRA & Me ha hecho sentir parte del grupo. & 425 & 4.83 & 1.521 & -.516 & -.185 & .563 \\
\hline 27 & CPP & $\begin{array}{c}\text { Me ha ayudado a ser más responsable hacia mi } \\
\text { aprendizaje. }\end{array}$ & 425 & 5.04 & 1.412 & -.676 & .273 & .690 \\
\hline 28 & CPS & Me ha ayudado en la aceptación de mis errores. & 424 & 5.22 & 1.403 & -.792 & .464 & .665 \\
\hline 29 & INTRA & Me ha generado motivación para el aprendizaje. & 424 & 4.61 & 1.573 & -.305 & -.526 & .696 \\
\hline 30 & INTRA & Me ha hecho sentir bien. & 423 & 4.46 & 1.532 & -.253 & -.413 & .647 \\
\hline 31 & INTRA & Me ha hecho mejorar la autoestima. & 424 & 3.69 & 1.613 & -.060 & -.772 & .535 \\
\hline 32 & INTRA & Me ha permitido ser más asertivo/a. & 424 & 4.73 & 1.488 & -.445 & -.213 & .625 \\
\hline
\end{tabular}

*COG: Desarrollo cognitivo y metacognitivo; CPS: Habilidades y competencias sociales; CPP: Competencias profesionales futuras; INTRA: Desarrollo de aspectos socioafectivos e intrapersonales.

Es importante apuntar que la hipótesis de partida no se cumple totalmente puesto que se identifican algunos ítems con dobles cargas. Además, tras el análisis vemos como los factores teóricos concernientes a las habilidades y competencias sociales y a las competencias profesionales pasan a ser un único factor, pese a que la literatura los distingue como dos dimensiones teóricas. Algunos de los ítems de estas dos dimensiones teóricas han pasado a formar parte de los otros factores. En la discusión avanzamos posibles explicaciones al respecto.

\section{Análisis factorial confirmatorio}

Partiendo de la estructura de tres factores, los resultados del AFC corroboran la estructura factorial sugerida por el AFE. El modelo que tiene un mejor ajuste, tras ajustar los índices de modificación y las covarianzas residuales estandarizadas, queda compuesto por tres variables latentes y 20 indicadores: S$\mathrm{B} \chi^{2}=481.056(163) ; \mathrm{p}=.000 ; \mathrm{CFI}=.934 ; \mathrm{TLI}=.923$; $\mathrm{GFI}=0.895$; RMSEA $=.06$. Los umbrales tomados como medidas de ajuste de parsimonia siguen las sugerencias de Calvo (2017). En síntesis, si bien se partió de un instrumento con un banco de 32 ítems iniciales, tras la depuración ha quedado conformado por 20 ítems distribuidos en tres factores.

\section{Análisis de fiabilidad}

El análisis de fiabilidad de los 20 ítems arroja un valor para el coeficiente Alpha de Cronbach muy elevado $(\alpha=.942)$ y indica que las características 
Tabla 2. Matriz de componente rotado ${ }^{\mathrm{a}}$

\begin{tabular}{|c|c|c|c|c|}
\hline & \multirow{2}{*}{ Items } & \multicolumn{3}{|c|}{ Componente } \\
\hline & & 1 & 2 & 3 \\
\hline 11 & Entender los criterios de evaluación y realizar la tarea de acuerdo con estos. & .732 & & \\
\hline 12 & Tomar conciencia de los objetivos de la asignatura. & .724 & & \\
\hline 8 & Contrastar conocimientos previos con nuevos conocimientos de la asignatura. & .713 & & \\
\hline 6 & Integrar los conocimientos de la asignatura en mis conocimientos generales. & .712 & & \\
\hline 13 & Entender mejor las tareas realizadas. & .682 & & \\
\hline 14 & Entender mejor tareas futuras. & .631 & & \\
\hline 7 & Comparar mi trabajo con el de los demás compañeros de clase. & .622 & & \\
\hline 10 & Aclarar dudas que tenía sobre la asignatura. & 617 & & \\
\hline 2 & Me ha hecho dar más valor a las tareas de la asignatura. & .573 & .503 & \\
\hline 4 & Es una estrategia de aprendizaje útil para mí. & .562 & & \\
\hline 1 & Ha sido útil para la mejora de las tareas de mi grupo. & .547 & & \\
\hline 24 & Ver la importancia de utilizar diferentes estrategias de evaluación para evaluar diferentes tareas. & .519 & & .498 \\
\hline 22 & Evaluar mejor mi trabajo. & .492 & & \\
\hline 31 & Me ha hecho mejorar la autoestima. & & .800 & \\
\hline 29 & Me ha generado motivación para el aprendizaje. & & .727 & \\
\hline 30 & Me ha hecho sentir bien. & & .725 & \\
\hline 3 & Ha aumentado mi confianza en los demás compañeros de equipo. & & .711 & \\
\hline 26 & Me ha hecho sentir parte del grupo. & & .710 & \\
\hline 5 & Ha mejorado mi aceptación por parte de los compañeros de grupo. & & 680 & \\
\hline 32 & Me ha permitido ser más asertivo/a. & & .654 & \\
\hline 27 & Me ha ayudado a ser más responsable hacia mi aprendizaje. & & .636 & \\
\hline 28 & Me ha ayudado en la aceptación de mis errores. & & .616 & \\
\hline 19 & Mejorar la capacidad de redactar mi discurso. & & & .760 \\
\hline 17 & Mejorar la capacidad de argumentación. & & & .749 \\
\hline 21 & Tomar conciencia de la responsabilidad de evaluar a otros. & & & .730 \\
\hline 20 & Evaluar mejor el trabajo de los compañeros. & & & .713 \\
\hline 18 & Mejorar la comunicación con los compañeros de grupo. & & .508 & .583 \\
\hline 15 & Mejorar mi competencia de trabajo en equipo. & & & .575 \\
\hline 16 & Aprender más activamente. & .465 & & .542 \\
\hline 9 & Mejorar mi capacidad comunicativa. & & .459 & .536 \\
\hline 23 & Mejorar la planificación de mis tareas. & & & .519 \\
\hline
\end{tabular}

Método de extracción: análisis de componentes principales.

Método de rotación: Varimax con normalización Kaiser.

a. La rotación ha convergido en 6 iteraciones.

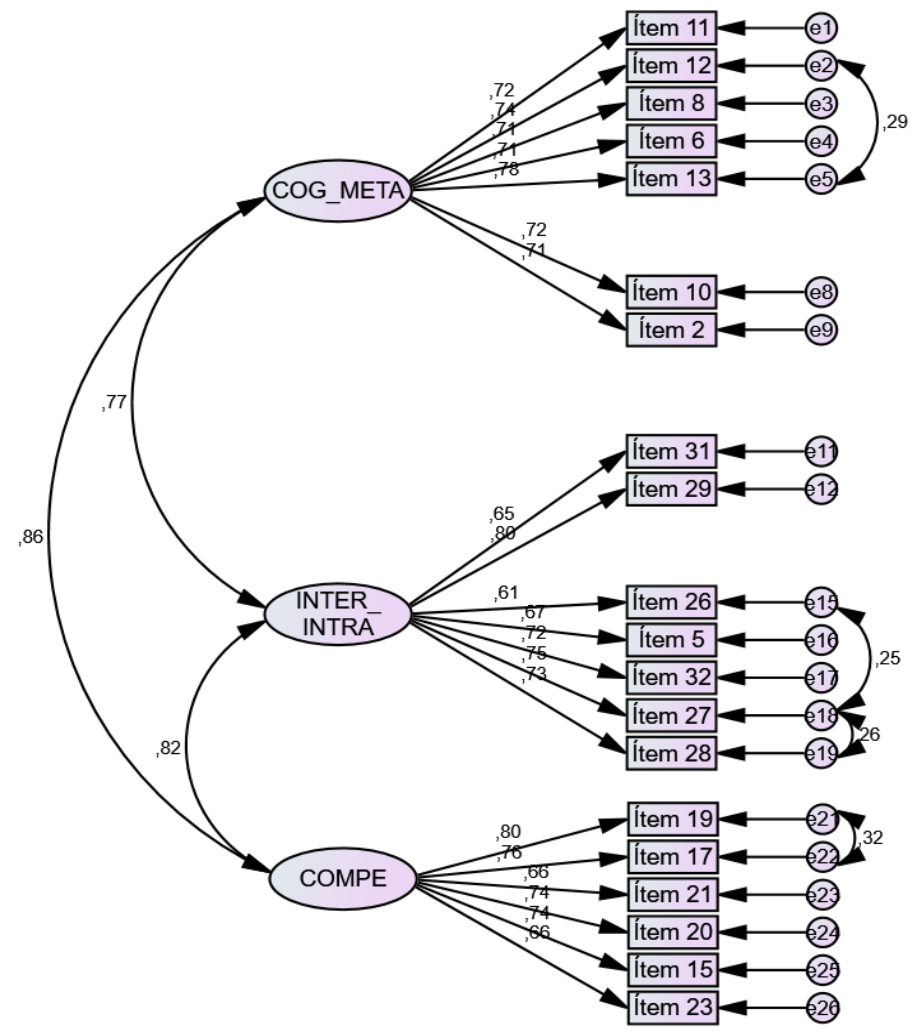

Figura 1. Estructura de factores y valores estandarizados para el modelo 
Tabla 3. Fiabilidad de las subescalas del

\begin{tabular}{lcc}
\multicolumn{3}{c}{ cuestionario ("darfeedback") } \\
\hline Subescalas & $\begin{array}{c}\mathrm{N} \mathrm{de} \\
\text { elementos }\end{array}$ & $\alpha$ Cronbach \\
\hline $\begin{array}{l}\text { Desarrollo cognitivo y } \\
\text { metacognitivo }\end{array}$ & 7 & .888 \\
$\begin{array}{l}\text { Desarrollo intrapersonal e } \\
\text { interpersonal }\end{array}$ & 7 & .876 \\
$\begin{array}{l}\text { Desarrollo competencial } \\
\text { Total }\end{array}$ & 6 & .871 \\
\hline
\end{tabular}

psicométricas del cuestionario son buenas. La fiabilidad de las subescalas se concreta en la Tabla 3 y como se puede apreciar los valores por subescalas son también muy buenos.

\section{Discusión}

El feedback emerge como un importante proceso en la evaluación orientada al aprendizaje que tiene lugar en la Educación Superior (Black \& Wiliam, 1998; Gibbs \& Simpson, 2002; Hattie, 1987), pero sigue siendo una estrategia débilmente articulada, especialmente, cuando reparamos en la evaluación entre iguales (Winstone et al., 2017). A través de instrumentos como el que se ha diseñado y validado se puede favorecer la reflexión y el conocimiento en torno a la contribución que dar feedback hace al aprendizaje en contextos de evaluación entre iguales.

Los resultados obtenidos mediante la estimación de la estructura de factores del modelo propuesto disponen de una adecuada bondad de ajuste y parsimonia, sosteniendo la idoneidad de los indicadores seleccionados en la medición de los constructos de interés del cuestionario. Por eso, su aplicación puede tener lugar en un contexto universitario en dónde se incorpore el feedback entre iguales para mejorar los aprendizajes de los estudiantes y para desarrollar competencias en ellos. Su uso sirve al logro de datos significativos sobre los resultados que se derivan de la evaluación entre iguales y desde la óptica del estudiante universitario.

La aportación más destacada del cuestionario tiene que ver con las dimensiones de análisis que lo integran. Este estudio ha investigado la estructura de las dimensiones teóricas sustentadas por la literatura (Panadero et al., 2014; Panadero, Jonsson, \& Strijbos, 2016b; Topping, 1998), y ha permitido corroborar tres enfoques integrados en el instrumento para analizar el efecto del feedback entre iguales en el aprendizaje y el desarrollo competencial en Educación Superior. Si bien, en el diseño del instrumento se ha partido de la distinción entre habilidades y competencias sociales, y competencias profesionales, los resultados arrojan una única dimensión que contiene ítems de ambos factores. Probablemente esto pueda explicarse porque si bien la literatura ha hecho esfuerzos para diferenciar entre aquellos procesos y habilidades que pueden trabajarse en el presente académico -nos referimos a las competencias y habilidades sociales-, la incorporación y adquisición de estos procesos son los que darían después lugar al desarrollo completo de las competencias para el futuro profesional. Constatamos así que dar feedback permite adquirir competencias (mayor autonomía en el proceso de aprendizaje, desarrollo del pensamiento crítico, seguridad y negociación del propio trabajo, etc.), siendo esto gradualmente extensible a otros ámbitos (social, laboral, profesional, etc.).

Por otra parte, observamos también que varios ítems de estas dimensiones teóricas competenciales saturan en el factor de desarrollo cognitivo y metacognitivo. La adquisición competencial en los estudiantes no se consigue de forma espontánea, sino que exige un trabajo sistemático y un importante grado de metacognición, entendido como consciencia clara de determinados procesos (Mateo, 2007) que permiten a posteriori alcanzar niveles de dominio más altos de una determinada competencia. Seguramente por ello varios ítems de las competencias sociales que implican regulación, monitorización y capacidad de iniciativa han quedado agrupados bajo el factor de desarrollo metacognitivo.

La configuración de estas tres categorías de análisis demuestra la naturaleza social del aprendizaje en el sentido ya señalado por Vygotsky. El aprendizaje se construye a través de la interacción entre los agentes implicados y el proceso de evaluación entre iguales creando el contexto propicio para que se active una serie importante de procesos de índole cognitiva, motivacional y emocional (Panadero, 2016) con impacto en el aprendizaje tanto de las personas que ofrecen el feedback como de aquellas que lo 
reciben (van Gennip, Segers, \& Tillema, 2009). Es una de las razones por las cuales el feedback entre iguales se considera una de las estrategias fundamentales de la evaluación para el aprendizaje (Panadero \& Alonso-Tapia, 2013). Dotar al estudiante de poder en el proceso de evaluación implica una multitud de factores sociales y humanos que necesitan considerarse y que convergen en la importancia de éste sobre la producción de pensamientos, acciones y emociones como resultado de la interacción entre los agentes implicados (Dixon, Hawe, \& Parr, 2011).

De una parte, la configuración de las dimensiones en tres categorías fundamentales que tienen que ver con la cognición y metacognición, aspectos interpersonales e interpersonales y las competencias, refleja la naturaleza fundamentalmente social del aprendizaje. De otra parte, la redistribución de los ítems vinculados con la percepción que se tiene acerca de la evaluación y el rol de las diferentes estrategias de evaluación en las categorías cognitivas e interpersonales son una evidencia que los estudiantes perciben la construcción de sus competencias profesionales directamente vinculada a las competencias de índole cognitivo, afectivo e interpersonal (Cano \& Fernández, 2016).

Igualmente, aun cuando en los últimos años se ha venido prestando una atención mayor tanto al desarrollo cognitivo y metacognitivo como a las competencias sociales y profesionales (Zheng, Cui, Li, \& Huang, 2018), también es cierto que los aspectos socioafectivos e intrapersonales han parecido caer en el olvido por cuanto se refiere a las oportunidades de aprendizaje que traen consigo sobre el feedback entre iguales (Panadero, 2016). Son muestra de ello los cuestionarios que, principalmente, se fijan en aprender de los errores, aprender a aprender y en competencias como la comunicación, el trabajo en equipo, la colaboración, el liderazgo o la innovación (Alcañiz, Chuliá, Riera, \& Santolino, 2015; Gómez \& Quesada, 2017; Santana-Bonilla et al., 2017). Se trata de instrumentos que van en la dirección de la satisfacción del estudiante con las experiencias desarrolladas, pero sobre todo de la adquisición de conocimientos para mejorar las distintas tareas académicas que se ejecutan. Lo mismo sucede con otro tipo de instrumentos como los cuestionarios de preguntas abiertas (VeraCazorla, 2017) o los grupos de discusión para evaluar entre iguales (Hortigüela \& Pérez, 2016). En todos los casos se echan en falta los aspectos socioafectivos e intrapersonales $\mathrm{y}$, por consiguiente, en nuestro trabajo estamos más próximos a propuestas como las de Strijbos, PatEl y Narciss (2010) que, implementadas en otras etapas educativas, consideran la afectividad como una dimensión imprescindible en el feedback entre iguales.

Sin embargo, el instrumento elaborado puede ser complementario de estos y también de otros que ponen el énfasis en el feedback recibido por los estudiantes. En este punto, cabe destacar que este estudio aporta conocimiento acerca del valor que tiene el feedback que los estudiantes ofrecen en un contexto de evaluación entre iguales y el impacto que éste logra en el desarrollo de competencias de los estudiantes (Ion, SánchezMartí, et al., 2018). Podemos concluir, en este sentido, que es un instrumento válido, interesado en los procesos de aprendizaje que se derivan de dar feedback y adaptado al ámbito de conocimiento de los estudiantes universitarios de las Ciencias de la Educación.

Por último, sobresale el doble propósito formativo y formador que en sí mismo tiene el cuestionario, porque no se limita a la simple identificación de dificultades, sino que asimismo ayuda a la mejor comprensión de todo aquello que se persigue con el feedback entre iguales. Esto invita al optimismo en el instante que se propicia vislumbrar aquello que se desearía sucediera a partir de la reflexión sobre los propios aprendizajes y las formas de alcanzar un mejor desarrollo académico, como han apuntado también otros trabajos (García et al., 2016; Gorostiaga, Balluerka, Ulacia, \& Aliri, 2018).

\section{Agradecimientos}

A la Oficina de Calidad Docente de la Universitat Autònoma de Barcelona por su apoyo económico en la realización de los dos proyectos que enmarcan esta contribución: "Estrategias de evaluación entre iguales y el feedback para la mejora de competencias" (2016/17) y "Mejorar la evaluación individualizada de los proyectos grupales mediante estrategias de evaluación entre iguales y feedback" (2017/18). 


\section{Referencias}

Ajjawi, R., \& Boud, D. (2018). Examining the nature and effects of feedback dialogue. Assessment \& Evaluation in Higher Education. doi:10.1080/02602938.2018.1434128

Alcañiz, M., Chuliá, H., Riera, C., \& Santolino, M. (2015). Evaluación formativa entre iguales: una experiencia de mejora competencial en estudiantes de Estadística. @itic Revista d'Innovació Educativa, 15, 4654.

Alexander, P. (2006). Psychology in Learning and Instruction. Ohio: Pearson Merril Prentice Hall.

Alonso, J. (2000). Motivar para el aprendizaje. Teorías y estrategias. Barcelona: Edebé.

Black, P., \& Wiliam, D. (1998). Assessment and classroom learning. Assessment in Education: Principles, Policy \& Practice, 5(1), 7-74. doi:10.1080/0969595980050102

Boud, D., \& Falchikov, N. (2006). Aligning assessment with long term learning. Assessment \& Evaluation in Higher Education, 31(4), 399-413. doi:10.1080/02602930600679050

Cabrera, N., \& Mayordomo, R. M. (Eds.). (2016). El feedback formativo en la universidad. Experiencias con el uso de la tecnología. Barcelona: LMI.

Calvo, C. (2017). Análisis de la invarianza factorias y causal con AMOS. (J.-P. Lévy \& B. Larraz, Eds.). Valencia: ADD Editorial.

Cano, E. (2014). Análisis de las investigaciones sobre feedback: Aportes para su mejora en el marco del EEES. Bordón. Revista de Pedagogía, 66(4), 9-24. doi:10.13042/Bordon.2014.66402

Cano, E., \& Fernández, M. (2016). Evaluación por competencias: La perspectiva de las primeras promociones de graduados. Barcelona: Octaedro.

Cano, E., \& Pons, L. (2019). Peer assessment as a pedagogical strategy to develop preservice teachers' self-regulation capacity. En AERA Annual Meeting. Toronto, Canada: American Educational Research Association (AERA).

Carless, D. (2015). La confianza: Facilitar el feedback dialógico. En D. Boud \& E. Molloy (Eds.), El feedback en educación superior y profesional. Comprenderlo y hacerlo bien (pp. 115-129). Madrid: Narcea.

Carless, D. (2016). Diseñar el feedback para promover el diálogo. En N. Cabrera \& R. M. Mayordomo (Eds.), El feedback formativo en la universidad. Experiencias con el uso de la tecnologia (pp. 13-30). Barcelona: LMI.

Carless, D., \& Boud, D. (2018). The development of student feedback literacy: Enabling uptake of feedback. Assessment \& Evaluation in Higher Education. doi:10.1080/02602938.2018.1463354

Carless, D., \& Chan, K. K. H. (2017). Managing dialogic use of exemplars. Assessment \& Evaluation in Higher Education, 42(6), 930941. doi:10.1080/02602938.2016.1211246

Crichton, H., \& Valdera Gil, F. (2015). Student teachers' perceptions of feedback as an aid to reflection for developing effective practice in the classroom. European Journal of Teacher Education, 38(4), 512-524. doi:10.1080/02619768.2015.1056911

Dawson, P., Henderson, M., Mahoney, P., Phillips, M., Ryan, T., Boud, D., \& Molloy, E. (2018). What makes for effective feedback: Staff and student perspectives. Assessment \& Evaluation in Higher Education. doi:10.1080/02602938.2018.1467877

Dixon, H. R., Hawe, E., \& Parr, J. (2011). Enacting assessment for learning: The beliefs practice nexus. Assessment in Education: Principles, Policy \& Practice, 18(4), 365-379. doi:10.1080/0969594X.2010.526587

Espasa, A. (2009). El feedback en el marc de la regulació de l'aprenentatge: Caracteritizació $i$ anàlisi en un entorn formatiu en línia. Universitat Oberta de Catalunya. Recuperado de https://www.tdx.cat/handle/10803/9128

Espasa, A., Guasch, T., Mayordomo, R. M., Martínez-Melo, M., \& Carless, D. (2018). A Dialogic Feedback Index measuring key aspects of feedback processes in online learning environments. Higher Education Research \& Development, 37(3), 499-513. doi:10.1080/07294360.2018.1430125

Ferrando, P. J., \& Anguiano-Carrasco, C. (2010). El análisis factorial como técnica de investigación en psicología. Papeles del Psicólogo, 31(1), 18-33.

Fletcher, R. B., Meyer, L. H., Anderson, H., Johnston, P., \& Rees, M. (2012). Faculty and students conceptions of assessment in Higher 
Education. Higher Education, 64(1), 119-133. doi:10.1007/s10734-011-9484-1

Gallego, B., Quesada, V., Gómez, M. A., \& Cubero, J. (2017). La evaluación y retroalimentación electrónica entre iguales para la autorregulación y el aprendizaje estratégico en la universidad: La percepción del alumnado. REDU. Revista de Docencia Universitaria, $\quad 15(1), \quad 127-146$. doi:10.4995/redu.2017.5991

Gan, Z., Nang, H., \& Mu, K. (2018). Trainee teachers' experiences of classroom feedback practices and their motivation to learn. Journal of Education for Teaching. doi:10.1080/02607476.2018.1450956

García, J. M., Inglés, C. J., Vicent, M., Gonzálevez, C., Pérez, A. M., \& San Martín, N. L. (2016). Validación de la Escala de Autoeficacia Percibida Específica de Situaciones Académicas en Chile y su relación con las estrategias de aprendizaje. Revista Iberoamericana de Diagnóstico y Evaluación - e Avaliação Psicológica, 41(1), 118-131.

Gibbs, G., \& Simpson, C. (2002). Does your assessment support your students' learning? London. Recuperado de http://isis.ku.dk/kurser/blob.aspx ?feltid=1577 44

Gielen, M., Peeters, E., Dochy, F., Onghena, P., \& Struyven, K. (2010). Scripting the role of assessor and assessee in peer assessment in a wiki environment: Impact on peer feedback quality and product improvement. Learning and Instruction, 20(4), 304-315. doi:10.1016/j.learninstruc.2009.08.007

Gómez, M. A. Á., \& Quesada, V. (2017). Coevaluación o evaluación compartida en el contexto universitario: La percepción del alumnado de primer curso. Revista Iberoamericana de Evaluación Educativa, 10(2), 9-30. doi:10.15366/riee2017.10.2.001

Gorostiaga, A., Balluerka, N., Ulacia, I., \& Aliri, J. (2018). Evaluación de la iniciativa personal en el ámbito educativo y su relación con la actitud emprendedora y el rendimiento académico. Revista Iberoamericana de Diagnóstico y Evaluación - e Avaliação Psicológica, $\quad 48(3), \quad$ 105-116. doi:doi:10.21865/RIDEP48.3.09
Harris, L. R., \& Brown, G. T. L. L. (2013). Opportunities and obstacles to consider when using peer- and self-assessment to improve student learning: Case studies into teachers' implementation. Teaching and Teacher Education, 36, 101-111. doi:10.1016/j.tate.2013.07.008

Hattie, J. A. (1987). Identifying the salient facets of a model of student learning: A synthesis of meta-analyses. International Journal of Educational Research, 11(2), 187-212.

Hortiguiela, D., \& Pérez, A. (2016). La evaluación entre iguales como herramienta para la mejora de la práctica docente. Opción, 7, 865-879.

Ibarra Sáiz, M. S., Gómez Rodríguez, G., Gómez Ruiz, M. Á., Ibarra-Sáiz, M., RodriguezGómez, G., \& Gómez-Ruíz, M. (2012). La evaluación entre iguales: Beneficios y estrategias para su práctica en la universidad. Revista de Educación, 359, 206-231. doi:10.4438/1988-592X-RE-2011-359-092

Ion, G., Cano, E., \& Fernández, M. (2017). Enhancing self-regulated learning through using written feedback in higher education. International Journal of Educational Research, 85, 1-10. doi:10.1016/j.ijer.2017.06.002

Ion, G., Sánchez-Martí, A., \& Agud, Í. (2018). Giving or receiving feedback: Which is more beneficial to student' learning? Assessment \& Evaluation in Higher Education, 44(1), 124138. doi:10.1080/02602938.2018.1484881

Ion, G., Sánchez Martí, A., \& Agud Morell, I. (2018). Giving or receiving feedback: Which is more beneficial to students' learning? Assessment \& Evaluation in Higher Education, $1-15$. doi:10.1080/02602938.2018.1484881

Johnson, D. W., \& Johnson, F. P. (1994). Joining together: Group theory and group skills (5. ${ }^{\mathrm{a}}$ ed.). Englewood Cliffs, NJ: Prentice Hall.

Kim, M. (2009). The impact of an elaborated assessee's role in peer assessment. Assessment \& Evaluation in Higher Education, 34(1), 105-114. doi:10.1080/02602930801955960

Li, L., Liu, X., \& Steckelberg, A. L. (2010). Assessor or assessee: How student learning improves by giving and receiving peer feedback. British Journal of Educational Technology, 41(3), 525-536. doi:10.1111/j.1467-8535.2009.00968.x 
Lloret-Segura, S., Ferreres-Traver, A., HernándezBaeza, A., \& Tomás-Marco, I. (2014). El análisis factorial exploratorio de los ítems: Una guía práctica, revisada y actualizada. Anales de Psicología, 30(3), 1151-1169. doi:10.6018/analesps.30.3.199361

López-Aguado, M. (2010). Diseño y análisis del Cuestionario de Estrategias de Trabajo Autónomo (CETA) para estudiantes universitarios. Revista de Psicodidáctica, 15(1), 77-99.

Marcoulides, G. A., \& Simkin, M. G. (2010). Evaluating student papers: The case for peer review. Journal of Education for Business, $67(2)$, 80-83. doi:10.1080/08832323.1991.10117521

Mateo, J. (2007). Interpretando la realidad, construyendo nuevas formas de conocimiento: El desarrollo competencial y su evaluación. Revista de Investigación Educativa, 25(2), 513-531.

Mauri, T., Ginesta, A., \& Rochera, M.-J. (2016). The use of feedback systems to improve collaborative text writing: A proposal for the higher education context. Innovations in Education and Teaching International, 53(4), 411-423. doi:10.1080/14703297.2014.961503

Molloy, E., \& Boud, D. (2015). Cambiar nuestra concepción del feedback. En D. Boud \& E. Molloy (Eds.), El feedback en educación superior y profesional. Comprenderlo y hacerlo bien (pp. 25-20). Madrid: Narcea.

Neugebauer, J., Ray, D. G., \& Sassenberg, K. (2016). When being worse helps: The influence of upward social comparisons and knowledge awareness on learner engagement and learning in peer-to-peer knowledge exchange. Learning and Instruction, 44, 4152. doi:10.1016/j.learninstruc.2016.02.007

Nicol, D., Thomson, A., \& Breslin, C. (2014). Rethinking feedback practices in Higher Education: A Peer review perspective. Assessment \& Evaluation in Higher Ed, 39(1), 102-111. doi:10.1080/02602938.2013.795518

Orsmond, P., \& Merry, S. (2013). The importance of self-assessment in students' use of tutors' feedback: A qualitative study of high and nonhigh achieving biology undergraduates. Assessment \& Evaluation in Higher Education, 38, 737-753. doi:10.1080/02602938.2012.697868

Ortego, M. del C., López, S., \& Álvarez, M. L. (2011). Ciencias Psicosociales I. Las habilidades sociales. Recuperado 2 de marzo de 2019, de https://ocw.unican.es/course/view.php?id=182 Panadero, E. (2016). Is it safe? Social, interpersonal, and human effects of peer assessment: A review and future directions. En G. T. L. Brown \& L. R. Harris (Eds.), Handbook of Social and Human Conditions in Assessment (pp. 247-266). New York: Routledge.

Panadero, E., \& Alonso-Tapia, J. (2013). Selfassessment: Theoretical and practical connotations. When it happens, how is it acquired and what to do to develop it in our students. Electronic Journal of Research in Educational Psychology, 11(2), 551-576. doi:10.14204/ejrep.30.12200

Panadero, E., Brown, G., \& Courtney, M. (2014). Teachers' reasons for using self-assessment: A survey self-report of Spanish teachers. Assessment in Education: Principles, Policy \& Practice, 21(4), 365-383. doi:10.1080/0969594X.2014.919247

Panadero, E., Jonsson, A., \& Strijbos, J. W. (2016a). Scaffolding self-regulated learning through self-assessment and peer assessment: Guidelines for classroom implementation. En D. Laveault \& L. Allal (Eds.), Assessment for Learning: Meeting the challenge of implementation (pp. 311-326). Boston, MA: Springer. doi:10.1007/978-3-319-39211-0_18

Panadero, E., Jonsson, A., \& Strijbos, J. W. (2016b). Scaffolding self-regulated learning through self-assessment and peer assessment: Guidelines for classroom implementation. En D. Lavezault \& L. Allal (Eds.), Assessment for Learning: Meeting the Challenge of Implementation (pp. 311-326). Boston, MA: Springer.

Paoloni, P. V., \& Rinaudo, M. C. (2009). Motivación, tareas académicas y procesos de feedback. Un estudio comparativo entre alumnos universitarios. Revista Electrónica de Motivación y Emoción, 11(31). Recuperado de

http://reme.uji.es/articulos/numero31/article9/t exto.html 
Pereira, D., Assunção, M., \& Niklasson, L. (2016). Assessment revisited: A review of research in assessment and evaluation in Higher Education. Assessment \& Evaluation in Higher Education, 41(7), 1008-1032. doi:10.1080/02602938.2015.1055233

Plana, D. (2016). El feedback efectiu personalitzat en entorns virtuals: Estudis des de la perspectiva estudiant-professor. Universitat Politècnica de Catalunya. Recuperado de https://upcommons.upc.edu/handle/2117/9812 8

Quinton, S., \& Smallbone, T. (2010). Feeding forward: Using feedback to promote student reflection and learning - a teaching model. Innovations in Education and Teaching International, $\quad 47(1), \quad$ 125-135. doi:10.1080/14703290903525911

Roth, A., Ogrin, S., \& Schmitz, B. (2015). Assessing self-regulated learning in higher education: A systematic literature review of self-report instruments. Educational Assessment, Evaluation and Accountability, 28(3), 225-250.

Rowe, A., Wood, L., \& Petocz, P. (2008). Engaging students: Student preferences for feedback. En Engaging Communities, 31st HERDSA Annual Conference, 1-4 julio (pp. 297-306).

San Martín, S., Jiménez, N., \& Jerónimo, E. (2016). La evaluación del alumnado universitario en el Espacio Europeo de Educación Superior. Aula Abierta, 44(1), 714.

Santana-Bonilla, P. J., Jiménez, F., Pintor, P., \& Hernández, V. M. (2017). Cómo implicar al alumnado en la evaluación formativa. El valor del feedback. En A. Vega \& D. Stendardi (Eds.), VII Jornadas de Innovación Educativa de la Universidad de La Laguna (pp. 169180). La Laguna: Universidad de La Laguna.

Stefani, L. A. J. (1994). Peer, self and tutor assessment: Relative reliabilities. Assessment \& Evaluation in Higher Education, 19(1), 6975.

Strijbos, J.-W., Pat-El, R. J., \& Narciss, S. (2010). Validation of a (peer) feedback perceptions questionnaire. En L. Dirckinck-Holmfeld, V. Hodgson, C. Jones, M. de Laat, D. McConnell, \& T. Ryberg (Eds.), Proceedings of the 7th International Conference on Networked Learning (pp. 378-386). Aalborg: Aalborg University.

Telio, S., Ajjawi, R., \& Regehr, G. (2015). The "Educational Alliance" as a framework for reconceptualising feedback in Medical Education. Academic Medicine, 90(5), 609614.

Topping, K. J. (1998). Peer assessment between students in colleges and universities. Review of Educational Research, 68(3), 249-276.

Topping, K. J. (2009). Peer Assessment. Theory into Practice, 48(1), 20-27.

Tsivitanidou, O. E. O. E., Zacharia, Z. C., \& Hovardas, T. (2011). Investigating secondary school students' unmediated peer assessment skills. Learning and Instruction, 21(4), 506519. doi:10.1016/j.learninstruc.2010.08.002

van Gennip, N. A. E., Segers, M. S. R., \& Tillema, H. H. (2009). Peer assessment for learning from a social perspective: The influence of interpersonal variables and structural features. Educational Research Review, 4(1), 41-54. doi:10.1016/j.edurev.2008.11.002

van Gennip, N. A. E., Segers, M. S. R., \& Tillema, H. H. (2010). Peer assessment as a collaborative learning activity: The role of interpersonal variables and conceptions. Learning and Instruction. doi:10.1016/j.learninstruc.2009.08.010

Vera-Cazorla, M. J. (2017). La evaluación entre iguales: estudio de caso en la Universidad de Las Palmas de Gran Canaria. Innovación Educativa, 27, 187-203.

Vygotsky, L. S. (1978). Mind in society: The development of higher psychological processes. Mind in Society The Development of Higher Psychological Processes. Cambridge, MA \& London, England: Harvard University Press.

Winstone, N. E., Nash, R. A., Parker, M., \& Rowntree, J. (2017). Supporting Learners' Agentic Engagement With Feedback: A Systematic Review and a Taxonomy of Recipience Processes. Educational Psychologist, 52(1), 17-37. doi:10.1080/00461520.2016.1207538

Zheng, L., Cui, P., Li, X., \& Huang, R. (2018). Synchronous Discussion between assessors 
and assessees in web-based peer assessment: Impact on writing performance, feedback quality, meta-cognitive awareness and selfefficacy. Assessment \& Evaluation in Higher Education, 43(3), 500-514. doi:10.1080/02602938.2017.1370533

Zhu, Q., \& Carless, D. (2018). Dialogue within peer feedback processes: Clarification and negotiation of meaning. Higher Education Research \& Development, 37(4), 883-897. doi:10.1080/07294360.2018.1446417 